\title{
CARTOGRAFIAR LOS AFECTOS Y LOS MOVIMIENTOS EN EL APRENDER CORPOREIZADO DE LOS DOCENTES
}

\author{
MAPEAMENTO DE AFETOS E MOVIMENTOS NA APRENDIZAGEM \\ CORPORIFICADA DOS PROFESSORES
}

\author{
MAPPING AFFECTIONS AND MOVEMENTS IN TEACHERS' EMBODIED \\ LEARNING
}

\author{
Sara Carrasco-Segovia*, Fernando Hernández-Hernández*
}

\begin{abstract}
Palabras clave: Corporeidad. Nuevos materialismos. Teoría de los afectos. Investigación postcualitativa.

Resumen: El cuerpo es el constructo central del ámbito de la Educación Física, pero, en los últimos años, la mirada sobre el cuerpo se ha expandido, en especial, a partir de los Nuevos Materialismos que han cuestionado y desplazado el sentido del cuerpo como realidad física y cultural a la noción de corporeidad. Este concepto vincula el cuerpo a lo relacional, va más allá de lo humano y expande las zonas de proximidad entre el cuerpo y el mundo. Esta perspectiva que diluye la idea del cuerpo como entidad que se cierra en sí misma, junto a la Teoría de los Afectos, ha llevado a cuestionar una noción de cuerpo inerte que espera pasivamente la imposición de una significación construida social y culturalmente por el sujeto. Desde este enfoque hemos indagado cómo un grupo de docentes de secundaria sitúa la corporeidad en sus trayectos en torno al aprender.
\end{abstract}

Palavras chave: Corporeidade. Novos materialismos. Teoria dos afeitos. Pesquisa pósqualitativa.

Keywords: Corporeality. New materialisms. Affects Theory. Post-qualitative research.

Resumo: O corpo é a construção central do campo da Educação Física, mas, nos últimos anos, o olhar sobre ele expandiu-se, sobretudo dos Novos Materialismos que questionaram e deslocaram o sentido do corpo como realidade física e cultural para a noção de corporeidade. Este conceito liga o corpo ao relacional, vai além do humano e expande as zonas de proximidade entre o corpo e o mundo. Esta perspectiva que dilui a ideia do corpo como entidade que se fecha em si mesma, juntamente com a Teoria dos Afetos, levou a questionar uma noção de corpo inerte que espera passivamente a imposição de um significado construído social e culturalmente pelo sujeito. A partir dessa abordagem, temos pesquisado como um grupo de professores do ensino secundário situa a corporeidade em seus caminhos em torno da aprendizagem.

Abstract: The body is the central construct in the field of Physical Education, but in the last few years, it has come under growing scrutiny, especially from the New Materialisms that have questioned and displaced the meaning of the body as a physical and cultural reality towards the notion of corporeality. This concept linking the body to the relational goes beyond the human and expands the areas of proximity between body and world. The perspective that dilutes the idea of the body as an entity that closes in on itself, along with the influence of the Theory of Affects, has led to challenging the notion of an inert body that passively waits for the imposition of a meaning socially and culturally constructed by the subject. Based on this approach, we have researched how a group of secondary school teachers situates corporeality in their learning paths.
* Universidad de Barcelona. Barcelona, España. E-mail: sara carrasco@ub.edu; fdohernandez@ub.edu

Recebido em: 24-07-2019 Aprovado em: 01-02-2020 Publicado em: 23-02-2020 


\section{INTRODUCCIÓN: SITUAR LA NOCIÓN DE CORPOREIDAD EN LOS TRAYECTOS DE APRENDER DE LOS DOCENTES}

En el grupo de investigación Esbrina (subjetividades, visualidades y entornos educativos contemporáneos, 2017SGR1248) prestamos atención a cómo los docentes dan cuenta de sus aprendizajes en diferentes escenarios (HERNÁNDEZHERNÁNDEZ; SANCHO-GIL; DOMINGO-COSCOLLOLA, 2018) y lo hacemos desde una perspectiva ecológica del aprender (COLL, 2013; ERSTAD; SEFTON-GREEN, 2013) que sitúa este complejo proceso más allá de lo cognitivo e introduce aspectos relacionales que tienen en cuenta las interacciones humanas y no humanas que se articulan en torno al aprender. En este camino hemos llegado a un aspecto que los colegas del campo de la Educación Física tienen como eje central en su proceso de investigación: el papel y el sentido del cuerpo en los procesos de aprendizaje (ANDREW; RICHARDS; LEVESQUE-BRISTOL, 2014; MACPHAIL, 2011).

En nuestro caso, el interés por indagar el papel del cuerpo en el proceso de aprender se vincula a la investigación "APREN-DO: Cómo aprenden los profesores: Implicaciones educativas y retos para afrontar el cambio social" (Ministerio de Economía y Competitividad/ EDU201570912-C2-1-R). En este estudio se invitó a un grupo de docentes de secundaria a generar cartografías visuales de sus escenarios de aprendizaje dentro y fuera de la escuela, así como a pensar sobre lo que podía constituir una fuente de conocimiento sobre su aprender en ese proceso de indagación. La cartografía actúa como una epistemología artística y una metodología narrativa de investigación que permite explorar fisuras, desplazamientos, formas de conocimiento y ensamblajes a través de los cuales los profesores dieron cuenta de sus trayectorias de aprender (PAULSTON; LIEBMAN, 1994; RUITENBERG, 2007).

Este proceso de investigación permitió aperturas a partir de, al menos, tres referentes clave:

a) el afecto que nos lleva a plantear el aprendizaje como un proceso que tiene lugar cuando el sujeto se sabe afectado (CVETKOVICH, 2012, p. 2);

b) las derivas post-cualitativas que nos ayudan a plantear cómo acceder a estos 'lugares' de aprendizaje más allá de los marcos preestablecidos, desde una investigación orientada en términos de procesos y relaciones rizomáticas (HERNÁNDEZ-HERNÁNDEZ; REVELLES BANAVENTE, 2019, p. 3); y

c) las cartografías, consideradas no sólo como un método visual de suscitar reflexiones, sino como un espacio en el que sustancias -cuerpos, cosas, textos, situaciones, ideas y formas de hacer- permanecen ensambladas (ULMER; KOROLJUNGBERG, 2015).

A partir de estos tres referentes afrontamos en este artículo el aprendizaje corporeizado de los docentes, entendiendo el cuerpo desde los Nuevos Materialismos, en especial, desde la noción corporeidad. Esta noción repiensa el cuerpo cuestionando su materialidad y como reacción a una noción de cuerpo que espera pasivamente la imposición de una significación como "construcción sociocultural" centrada únicamente en el sujeto (CLOUGH, 2009). 


\section{ESTADO DE LA CUESTIÓN. SITUAR EL LUGAR DEL CUERPO EN EL TRÁNSITO DESDE LA NOCIÓN DE CORPORALIDAD A LA DE CORPOREIDAD}

El cuerpo es el tema central en el campo de la Educación Física y también ha sido cuestión de interés las últimas décadas en las Ciencias sociales, en especial, desde la teoría social contemporánea del siglo XX. En este sentido, Martínez (2004) ha analizado algunos de los principales cambios que han operado sobre la imagen social del cuerpo desde una comprensión que difiere de la idea clásica del dualismo entre cuerpo-alma (espíritu) de la metafísica tradicional y que fue planteada por autores como Descartes. Martínez (2004) hace notar el descuido que ha tenido la teoría social clásica con el cuerpo. Descuido que, según Turner (1994), se debe a dos causas principales:

a) al legado del dualismo cartesiano, y

b) centrarse únicamente en el ser humano como creador de signos y significados dejando de lado el aspecto social del cuerpo. Darse cuenta de este olvido provocó un cambio en la noción que se tenía del cuerpo hasta esos momentos: una organización fisiológica que promovía la diferencia entre cuerpo y alma como entidades separadas. Merleau-Ponty (1973) fue uno de los autores que desarrolló la crítica de la primacía ontológica del dualismo mediante una reubicación del cuerpo en sus posibilidades experienciales, dando fundamento a la afirmación según la cual el ser humano establece una manera de ser cuerpo. En relación con esta posición, Le Breton (2002), Turner (1994) y otros han abordado el estudio del cuerpo desde su implicación en la vida social, en las relaciones entre los cuerpos y en la experiencia encarnada (embody experience). Este último concepto se vincula a la idea de que el cuerpo es el soporte de nuestras prácticas sociales y que está moldeado por ellas (RODRÍQUEZ, 2014). De esta manera el cuerpo de las teorías sociales pasa a concebirse como portador de fuerzas sociales.

A partir de aquí se ha promovido una noción del cuerpo que centra su comprensión en el sujeto (desde su biografía vinculada a lo social) y en las fuerzas sociales humanas con las que se relaciona y le confieren su sentido. Así, el cuerpo deviene en una construcción social que no se da de manera natural, sino que se enmarca culturalmente en un momento histórico, social y político, al tiempo que constituye y participa de nuestra vida social ampliando sus límites físicos, orgánicos, simbólicos y relacionales. Desde esta idea, Le Breton (2002) y Turner (1994) llegaron a una noción social del cuerpo en la que pasa a ser visto como algo que va más allá de lo propiamente físico y se concibe como parte esencial y constitutivamente subjetiva de la persona: la corporalidad. Lo que significa, como señala MerleauPonty, que "[...] no estoy delante de mi cuerpo, no estoy en mi cuerpo, sino que soy mi cuerpo" (1945, p. 175).

Para Esteban (2004), el cuerpo desde estas teorías sociales deja de lado algunos de sus múltiples atributos y olvida su materialidad dentro de una articulación en la que operan fuerzas que no son únicamente humanas, sino también materiales, no humanas y más que humanas. A partir de este cuestionamiento, Domínguez y Lara (2014) consideran que las teorías sociales desarrollaron aproximaciones al margen 
del cuerpo durante toda la segunda mitad del siglo XX y cuando se dirigieron hacia él, se trató del cuerpo del sujeto y, sobre todo, del sujeto de conocimiento (CLOUGH, 2009). Los cuerpos son considerados entes inertes que esperan pasivamente la imposición de un significado a través de la construcción social y cultural. De aquí que Lara y Domínguez (2013) propongan ir hacia las contribuciones del Giro Afectivo, que destaca el papel del movimiento hacia y desde el cuerpo como generador de afectos y cuestiona el privilegio del estudio del cuerpo basado en el significado y el discurso. Este movimiento promueve una noción sobre el cuerpo que replantea su materialidad, considerándolo como una fuerza dinámica y poseedora de potencial para su propia organización.

Según Clough (2009), el cuerpo en la Teoría del Afecto es abordado por su especificidad biológica y por sus capacidades subindividuales, en contraste con los modelos construccionistas centrados en el sujeto y en la experiencia encarnada reducible únicamente a las estructuras sociales, dejando de lado sus múltiples atributos y olvidando su materialidad dentro de una articulación performativa que se mueve entre diferentes fuerzas. El afecto, en la tradición spinoziana (SPINOZA, 1980), es considerado como precedente directo del cuerpo sin la interferencia o las limitaciones de la conciencia y la representación (LARA, 2015). Por ello, las diversas perspectivas desarrolladas dentro de la Teoría del Afecto son cruciales para comprender las nuevas lógicas y relaciones del cuerpo y de los afectos, revelándose como ruta para abordar el concepto de cuerpo y sus diversas trayectorias.

A partir de estos desplazamientos en el entendimiento del cuerpo, nos centramos en la noción de corporeidad (corpor[e]ality) acuñada por Grosz (1987, 1994), y su aportación al estudio del cuerpo desde los Nuevos materialismos, para pensar cómo se articula el aprendizaje corporeizado de los docentes.

La noción de corporeidad media una noción de cuerpo que ya no es concebido como una construcción social que sólo depende de las fuerzas humanas, sino como un territorio experiencial, como una zona de indecisión, indeterminación, de conexiones, movimientos y relaciones (BARAD, 2012; ROGOWSKA-STANGRET, 2017). Esta comprensión del cuerpo se relaciona con lo que Grosz $(1987,1994)$ llamó corporeidad ${ }^{1}$, que desplaza el cuerpo hacia lo inhumano, lo más que humano, lo más allá de lo humano y se concentra en las zonas de proximidad entre el cuerpo y el mundo. "Corporeality thus embraces the outside of corporality — the inhuman dimension of the body, its animal, plant, inorganic, technological elsewhere - and subsequently changes the very concept of the body" (ROGOWSKA-STANGRET, 2017, p. 63).

El movimiento desde el concepto corporalidad (corporality) - parte constitutivamente subjetiva de la persona- al de corporeidad (corpor[e]ality), se genera al considerar la dimensión incorpórea del cuerpo, al pensarlo hacia lo que está más allá de sí mismo -hasta ahora pensado desde el sujeto humano-, hacia "su otro lugar" (elsewhere), hacia lo inhumano. El cuerpo entendido de este modo se abraza al exterior, al mundo y a lo que lo rodea, rompiendo de esta manera con

1 La autora marca la diferencia entre corporality y corporeality, agregando una "e" que marca el desplazamiento del cuerpo hacia esos otros lugares que se señalan en el artículo. 
la división dualista exterior-interior y cultura-naturaleza al pensar lo humano-animal y humano-inorgánico como oposiciones (ROGOSWKA-STRANGRET, 2017). Este acercamiento al cuerpo escapa de los poderes universalizadores y normalizadores, eludiendo trampas y peligros del pensamiento oposicional y de los enfoques estandarizados que favorecen los binarismos.

Para Rogoswka-Strangret (2017), esta reconceptualización de la corporalidad de Grosz (2004) tiene consecuencias cruciales para el debate en torno al cuerpo. El paso de corporalidad a corporeidad desplaza la corporalidad hacia su otra parte inhumana y captura las conexiones intrínsecas que el cuerpo tiene con/en el mundo (GROSZ, 2004, p. 2). La corporeidad le otorga importancia a una agencia relacional que se desprende en aquello más que humano dentro del cuerpo en sí mismo; esto produce una relación entre el fuera y el dentro de lo humano que actúa como un continuo y no como una oposición. También presta atención no sólo al cuerpo inmerso en el mundo, sino a los cuerpos como (re)configuraciones dinámicas del mundo, prácticas/intra-acciones/interpretaciones agenciales (BARAD, 2003, p. 816). Además, la transición de la corporalidad a la corporeidad cambia el enfoque de la investigación teórica del "ser humano" hacia la "realidad" (ROGOSWKA-STRANGRET, 2017, p. 62). Finalmente, este cambio priva al sujeto humano y al cuerpo humano de su posición privilegiada de control, dominación y poder sobre lo no humano.

\section{EL PROBLEMA DE LA INVESTIGACIÓN. ACERCARSE A LA CORPOREALIDAD DE LOS DOCENTES DESDE LAS CARTOGRAFÍAS SOBRE SUS TRAYECTORIAS DE APRENDIZAJE}

Dar cuenta del modo de acercarnos a los movimientos de los docentes sobre sus trayectos de dónde y cómo aprenden, requiere replantear el sentido de cómo se suele atender una aproximación metodológica. Desde una posición post-cualitativa (LATHER, 2013; LATHER; ST. PIERRE, 2013; MAZZEI, 2013) no se trata de diseñar una situación que permita generar unos datos para luego analizarlos y dar cuenta de sus posibles sentidos de acuerdo con las decisiones previamente establecidas. Se trata de un sentido de investigar que no persigue encontrar y desvelar lo que está 'ahí fuera', pues se reconoce que el afuera está enredado con la mirada de quien investiga. Esto hace que el proceso de indagación (inquiry) sea un recorrido relacional donde se está atento no a lo que se espera encontrar, sino a lo que (nos) va aconteciendo en un proceso que no es lineal, sino repleto de bifurcaciones, dudas y lugares de no saber (ATKINSON, 2011; 2015).

Desde estas premisas daremos cuenta en los siguientes párrafos de lo que nos permiten pensar las cartografías, y lo que han dicho sobre ellas los docentes, a partir de la noción de corporealidad y de cómo las experiencias y lugares de aprender resuenan desde este concepto. Lo hacemos como tentativa de respuesta a la invitación que nos hace Masny (2016, p. 666), "[...] to experiment with post-qualitative research and to think data in terms of problems, questions, and concept creation". Las cartografías fueron realizadas por los docentes para mapear el "cómo" aprenden y no el "qué" aprenden, centrando la importancia en el proceso y no en el resultado del aprender. Las cartografías se configuran como un método desencadenante 
que permite a los docentes hablar sobre lo que para ellos era aprender, dónde aprendían y cómo transitaban dentro y fuera de las escuelas. Las cartografías se plantean, como una estrategia visual que se cruza con el texto y la palabra (ULMER; KORO-LJUNGBERG, 2015) y que contribuyen a indagar y ampliar los sentidos del aprender de los docentes. En las cartografías hemos prestado especial atención a la corporeidad de los docentes en sus trayectorias de aprendizaje.

\section{MÉTODOLOGIA. LOS MOMENTOS CARTOGRAFÍCOS PARA ACERCARSE A LOS PROCESOS DE APRENDER DE LOS DOCENTES}

El inicio de la investigación fue un "taller" en el que los investigadores realizaron sus cartografías de aprendizaje. En esta actividad se hizo patente que se entraba en un terreno de no saber; que las cartografías suponían entrecruzar imágenes, palabras y relatos que daban cuenta de recorridos biográficos, pero también de deseos que se articulaban entre lo insinuado y lo no dicho. Todo ello, en un marco que colocaba a los investigadores ante la dificultad de afrontar un problema -el de cartografiar-, que introducía a docentes e investigadores en un territorio inexplorado: la narración visual de la experiencia de aprender.

Con este bagaje se conectó, después de realizar un estudio piloto con dos docentes, con tres profesoras con las que se había tenidos contactos previos y a las que se les propuso participar en la investigación. Pero lo imprevisto irrumpió a través de la propuesta que ellas plantearon: “¿por qué en lugar de hacerlo de manera individual no venís a nuestro instituto y lo hacemos con todos los profesores que quieran participar?".

A los centros fuimos en dos ocasiones. En la primera, las situaciones que aparecieron, que no se podían prever de antemano fueron las siguientes. En uno de los institutos, el grupo (9 mujeres y 2 hombres) estaba afectado por la intervención de un padre en contra de una docente; esta situación generaba un malestar que les hizo dudar si participar o no de la propuesta que les habíamos hecho. En otro, quienes participaron (5 mujeres y 2 hombres), mantenían vínculos al compartir un proyecto de integración de lenguas y habían respondido a la invitación por el interés en quién había sido nuestro contacto inicial. En el tercero, vinieron 6 mujeres y 1 hombre. Una de ellas era la directora, con quien inicialmente habíamos conectado, y el resto compartía con ella su interés por la mejora del instituto. Durante la primera visita, después de presentarse y compartir su motivación por participar en la investigación, comenzaron a realizar las cartografías acompañados por un investigador, quien no pautaba sino acompañaba en las dudas que podían surgir. Una vez finalizadas las cartografías, cada docente hizo un relato sobre las decisiones tomadas y el contenido de las mismas. Esta narración fue filmada para poder trabajar sobre su transcripción.

En la segunda, cuatro meses después, compartimos lo que nos habían hecho pensar las cartografías y las conversaciones mantenidas, abriendo así un nuevo modo de relación en el que intercambiamos cómo a ellas y a nosotros nos había afectado la experiencia compartida. 
En paralelo, 3 profesoras vinieron a la universidad para realizar sus cartografías. De esta manera, 24 mujeres y 6 hombres nos regalaron sus historias y su tiempo.

\section{EL ANÁLISIS COMO UNA FORMA DE DIÁLOGO Y DE PENSAR A PARTIR DE CONCEPTOS}

Uno los aspectos que pone en cuestión la perspectiva post-cualitativa es el sentido del análisis y de la propia noción de "datos". Si el movimiento que agita la investigación es desde la inmanencia, hay que mantener ese fluir en relación con lo que se produce en la trama de encuentros que se van generando durante la investigación. Esto hace que el análisis no se configure como una aplicación de una fórmula preestablecida para encontrar aquello que ya hemos decidido de antemano. Por eso, en lugar de aplicar una estrategia para desmenuzar las cartografías y lo que los docentes dijeron sobre ellas, y luego agruparlas por temas, nuestra postura parte del reconocimiento y la potencialidad del "no saber" (ROGOFF, 2006). Así, buscamos un nuevo tipo de objeto de investigación, "pulled out of shape by its framings" y, lo que es igualmente importante, "framings pulled out of shape by the object" (LATHER, 2013 , p. 639). Con este fin, las cartografías, los relatos de las profesoras en torno a ellas y nuestras notas de campo fueron reunidas para producir una conversación que se plasma en el proceso de escritura de la investigación. Esto nos permitió abrirnos a "unexpected readings of and listening to materials in what might be termed 'fractal analysis"” (LATHER, 2016, p. 127).

En este proceso no intentamos analizar las cartografías, sino movernos a través de ellas y señalar algunos conceptos que emergen en relación con el aprendizaje y el cuerpo. Vamos a detenernos en la relación entre dos de ellos (afectos y corporealidad). Para ello, nos centramos en una docente que nos permite explorar e ilustrar algunos de estos conceptos y desarrollar el argumento central de este artículo: las relaciones entre los cuerpos (corporeidades), los lugares, objetos y procesos de aprender.

\subsection{RESULTADOS. APRENDER COMO TRAMA ENTRE EL CUERPO Y LOS AFECTOS}

Para ilustrar nuestra aproximación a los movimientos del cuerpo que posibilita realizar las cartografías, se ha elegido a una profesora con experiencia en la enseñanza de la Lengua y Literatura Castellana. La hemos escogido con el criterio de que su cartografía y su relato posibilitan explorar con nitidez los vínculos entre la corporeidad, los afectos y la experiencia de aprender. El procedimiento que hemos seguido para realizar este diálogo ha sido el siguiente. Primero hacemos una presentación de la profesora, a partir de lo que ella dice de sí misma y nuestras observaciones. A partir de aquí, entrecruzamos el contenido evocativo (HERNÁNDEZ-HERNÁNDEZ, 2008) de la cartografía con el relato que realiza la docente, poniéndolo en relación con los tres conceptos apuntados más arriba (experiencia de aprender, afectos y corporealidad), con lecturas que expanden los sentidos de lo dicho y con las resonancias que este recorrido provoca en nosotros. Todo ello, con la finalidad de explorar "las sensaciones, intensidades y texturas" (COLEMAN; RINGROSE, 2014, p.4) a través de las cuales 
se experimenta el aprendizaje y para comprender la multiplicidad de los mundos de aprendizaje de la docente y las cuestiones que plantean su cartografía, no como métodos que 'captan' estas realidades, sino que las convierten en realidad.

\section{Montse: Aprender, donde los movimientos del cuerpo y los afectos se enredan}

Montse (nombre anonimizado) es profesora de Literatura y Lengua Castellana con una extensa trayectoria en la que ha generado prácticas alternativas para el aprender de los jóvenes. Investiga sobre lo que hace en sus clases. Manifiesta que,

[...] eso me ha llevado a formar parte de un grupo de profesores de secundaria que nos reunimos una vez al mes, o mes y medio, para intercambiar experiencias y a veces invitamos a un experto en algún tema. Todo eso influye en la manera de dar clases y en nuestro tiempo libre ya que es por amor al arte, de manera que está todo muy relacionado. (Montse, 2017)

Además, para ella, "el mundo de la lectura, de la literatura es media vida",

[...] me encanta pasarme horas leyendo una novela por puro placer. Cuando estudiaba, trabajé en una biblioteca escolar y eso se refleja muchísimo en mi trabajo, ya que intento fomentar que mis alumnos se aficionen a la literatura y que la vivan. (Montse, 2017)

Figura 1 - Cartografía de Montse.

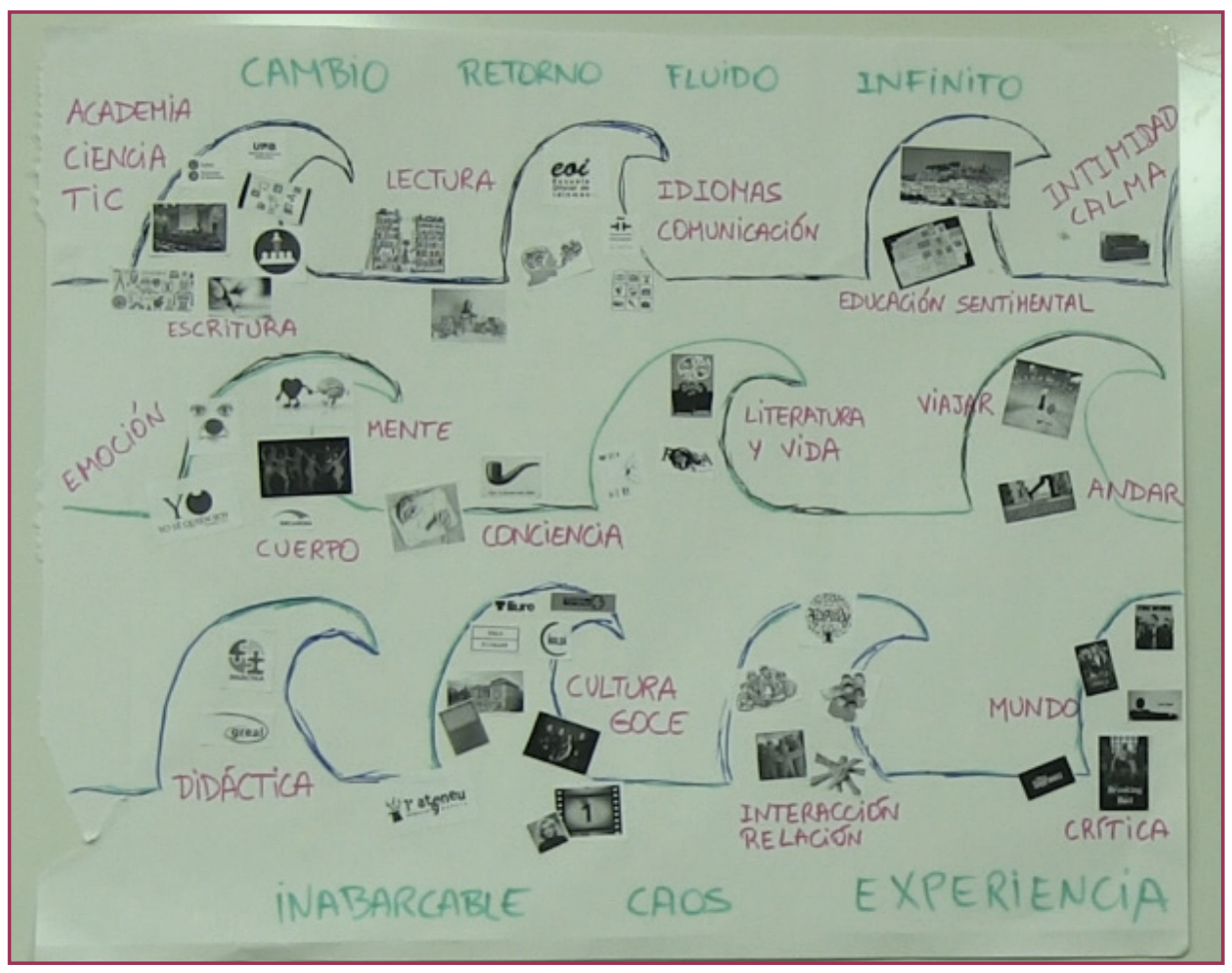

Foto de Alejandro Poblete.

La estructura de su cartografía (figura 1) toma la metáfora de las olas, para dar cuenta de lo "inabarcable de la experiencia" y del constante movimiento (el devenir) en el que transita su proceso de aprender. En estos movimientos el afecto se despliega en el flujo y reflujo, como el ciclo de la marea, y se transforma a sí mismo y transforma 
lo que le rodea y encuentra nuevos significados, aplicaciones y potencialidades a través de su uso [...] es una materialidad que siempre ha estado y está en proceso (BAKKO; MERZ, 2015, p. 8).

Para mí era muy difícil pensar en qué forma tiene que tener una metáfora del aprendizaje, ya que no tiene forma, es algo infinito, sin límites y bueno, no se acaba nunca [...] Por eso he puesto también lo del retorno, va y viene, y mis ámbitos de aprendizaje van y vienen todo el rato en mi vida, se dan muchos a la vez también. (Montse, 2017)

En el encuentro con Montse, el cuerpo aparece como apertura, como salida hacia otros espacios del conocer vinculados al crecimiento personal que contribuyen a replantear la dicotomía cartesiana.

\begin{abstract}
Hasta cierto momento de mi vida, digamos que tenía mucho que ver con lo académico, lo mental, hasta que empecé hacer un trabajo de crecimiento personal y de ver las soluciones en las formas de aprendizaje y que el cuerpo también es aprendizaje. El cuerpo también relacionado con lo físico, pero no solamente, sino también con lo emocional y que por ahí se puede crecer. (Montse, 2017)
\end{abstract}

Aquí irrumpe el afecto como manera de entender el mundo como un movimiento eterno entre cuerpos cambiantes donde las fuerzas invisibles posibilitan encuentros entre aquello corpóreo y fuerzas no solamente humanas (HICKEY-MOODY, 2016, p.260). Y en los afectos, el cuerpo está relacionado directamente con el movimiento y con el hacer (desde su dimensión física), y esto es fundamental para Montse en su experiencia de aprender. "Para mí, aprender es hacer y hacer, y haciendo cosas se aprende; con el movimiento, pero también con la tranquilidad, con la reflexión. Y a veces perder el tiempo también es sano." (Montse, 2017)

En este movimiento de un hacer en el que se aprende, los afectos, como señalan Bakko y Merz (2015, p. 8) aparecen como "[... ] una fuerza de indeterminación social que nos ofrece la oportunidad de mirar lo que es, imaginar lo que podría ser en su lugar, y comprender que este 'en su lugar' siempre está sucediendo".

\begin{abstract}
Empecé hacer un trabajo de crecimiento personal y de ver las soluciones en las formas de aprendizaje y que el cuerpo también es aprendizaje. El cuerpo relacionado con lo físico, pero también con lo emocional [...]. Esto tiene una vinculación directa con mis clases, pues de repente te das cuenta de que, a niños de 12 años, los tienes sentado durante seis horas, sentado entre 4 paredes. Yo les hago hacer improvisaciones, tener más en cuenta sus emociones, lo que pueden estar pasando, buscar también soluciones a sus necesidades y sensibilizarme un poco con sus necesidades. (Montse, 2017)
\end{abstract}

El espacio del aprender está aquí cruzado por el cuerpo, pero es un cuerpo cuya disponibilidad no sólo tiene que ver con el movimiento y la performatividad, sino también con la inmovilidad provocada por el espacio institucional. Es un cuerpo que es está siendo afectado y a su vez afecta los modos de aprender, desde su conexión con la experiencia y los afectos de los estudiantes y de Montse como profesora. De esta manera, los movimientos de aprender que se cruzan en Montse posibilitan, cuando los cuerpos entran en relación física y temporal (COLMAN, 2010, p. 13), que los afectos se movilicen, como "los procesos, fuerzas, poderes y expresiones de cambio [...] que en su combinación producen una [...] transformación en el cuerpo afectado" (COLMAN, 2010, p. 12). Algo que acontece porque "los afectos son devenires" (DELEUZE; GUATTARI, 2004, p. 256). El afecto [...] es una fuerza 
experiencial o una fuente de poder que, a través de encuentros y mezclas con otros cuerpos [...] queda envuelto por el afecto, convirtiéndose en una idea (COLMAN, 2010 , p. 13). La idea de un aprender encarnado y proyectado en movimientos de afecto.

Este movimiento se despliega en los viajes que, para Montse, son una experiencia de aprender que se corporiza en una forma de relacionarse con el mundo que luego lleva a la vida del aula, a la enseñanza de la literatura, al amor por la lectura con los estudiantes.

\begin{abstract}
Viajar es la manera de relacionarte con los demás y con el mundo. Viajar es enseñar una manera de mirar el mundo, de que aprendas con la vivencia y es la manera fundamental de aprender, porque es algo que se incorpora a ti y surge de ti [...] hacer una serie de actividades, ir al museo, recorrer la ciudad andando [...] la mejor manera de relacionarte con el mundo es tocar cada piedra. (Montse, 2017)
\end{abstract}

La noción de experiencia emerge aquí como algo primordial, pero también el aprendizaje se corresponde con la noción de desplazamiento, que no es sólo físico y desde su atributo móvil sino también con un cambio en el enfoque de la mirada. Este modo de aprender está cruzado por el cuerpo -está conectado con otros cuerpos y con otras situaciones- afectando y siendo afectado.

Montse, como la mayoría de las profesoras involucradas en la investigación, concibe el cuerpo como una característica clave en sus experiencias de aprender; está siempre presente incluso cuando lo esconden, consideran su pasividad y/o es invisibilizado. Esta presencia se configura en el aprender mediante movimientos afectivos "[...] donde los cuerpos pueden experimentarse y conectarse para ir más allá de sus propios límites" (ROGOWSKA-STANGRET, 2017, p. 60). Pero no es un cuerpo que actúa aislado y por sí mismo dentro de una constitución idealizada de lo que significa ser docente, sino un cuerpo que señala un enredo de relaciones entre todos aquellos elementos que forman parte de su proceso de aprender, con la disciplina, la espacialidad, la arquitectura, la geografía, lo humano, el autoconocimiento, el movimiento, el afecto... Esto acontece porque, como señala Barad (2007 en ARLANDER, 2014) el mundo es reconfigurado en un continuo de intra-acciones donde los elementos se combinan para la acción, no como el resultado de ella. En las intra-acciones los cuerpos que se crean, entonces, no existen como elementos individuales, sino que surgen a partir de la intra-acción (ARLANDER, 2014, p. 28).

\title{
6 CONCLUSIONES
}

En el recorrido que aquí se presenta se ha puesto el énfasis en la relación del cuerpo, entendido como cuerpo en relación -corporealidad- en los trayectos de aprendizaje de los docentes. Desde esta finalidad, la investigación posibilita pensar sobre cómo aprenden los docentes:

Los docentes aprenden en una trama de relaciones, en las que se vincula lo biográfico, lo corpóreo además de lo cognitivo.

Los docentes aprenden transitando desde el 'afuera' a la vida diaria de la escuela. En particular en las relaciones con los colegas y los estudiantes. 
Los docentes dan cuenta de una dimensión extensa de su aprendizaje en la vinculación que realizan con sus experiencias de vida de aprender.

Para los docentes aprender es una noción que se expande y es atravesada por el cuerpo, los afectos y las experiencias de aprender.

A partir de aquí se puede "pensar (el aprender) a través de los cuerpos" (ALAIMO, 2010, p. 2 en ROGOWSKA-STANGRET, 2017, p. 64) en tanto que la corporealidad es "constitutiva de lo que significa pensar y existir" (NARDINI, 2014, p. 23) y plantea "las raíces corporales del proceso de pensamiento" (BRAIDOTTI, 1991, p. 8 en NARDINI, 2014, p. 20). Unas raíces que tienen que ver con el movimiento inevitable de los cuerpos afectados en las intra-acciones con el entorno y con otros cuerpos (DE RIVA MAYORAL; RIVELLES BENAVENTE, 2019).

De esta manera, la cuestión parece clara: el afecto crea movimiento y, al mismo tiempo, el movimiento de un cuerpo posibilita acercarse a los afectos. Este movimiento posibilita aprender con sentido más allá de lo que encierra en el aprendizaje escolar.

\section{REFERENCIAS}

ALAIMO, Stacy. Bodily Natures: Science, Environment, and the Material Self. Bloomington: Indiana University, 2010.

ARLANDER, Annette. From interaction to intra-action in performing landscape. Artnodes, $\mathrm{n}$. 14, p. 26-34. 2014.

ATKINSON, Dennis. Art, Equality and Learning. Pedagogies Against the State. Rotterdam / Boston / Taipei: Sense, 2011.

ATKINSON, Dennis. The adventure of pedagogy, learning and the not-known. Subjectivity, v. 8, n. 1, p. 43-56, 2015.

BAKKO, Matthew; MERZ, Sibille. Toward an Affective Turn in Social Science Research? Theorising Affect, Rethinking Methods and $(\mathrm{Re})$ Envisioning the Social. Graduate Journal of Social Science, v.11, n.1, p. 7-14, Feb. 2015.

BARAD, Karen. Posthumanist Performativity: Toward an Understanding of How Matter Comes to Matter. Journal of Women in Culture and Society, v. 28, n.3, p. 801-831, 2003.

BARAD, Karen. Meeting the Universe Halfway: Quantum Physics and the Entanglement of Matter and Meaning. Durham: Duke University, 2007.

BARAD, Karen. Thinking with intra-action. In: JACKSON, Alecia y MAZZEI, Lisa (Eds.).

Thinking with theory in qualitative research. Viewing data across multiple perspectives. New York: Routledge, 2012. p 118-136.

BRAIDOTTI, Rossi. The Subject in Feminism. Hypatia. A Journal of Feminism Philosophy, v.6, n.2, p. 155-172, 1991. Disponível em: https://doi. org/10.1111/j.1527-2001.1991.tb01399.x Acesso em: 11 fev. 2020.

CLOUGH, Patricia. The new empiricism. Affect and Sociological method. European Journal of Social Theory, v.12, n.1, p.43-61, 2009. 
COLL, César. La educación formal en la nueva ecología del aprendizaje: Tendencias, retos y agendas de investigación. In: RODRÍGUEZ-ILLERA, José Luis. (comp). Aprendizaje y educación en la sociedad digital. Barcelona: Universitat de Barcelona, 2013. p. 156-170.

COLEMAN, Rebecca; RINGROSE, Jessica. Deleuze and research methodologies. Edinburgh: Edinburgh University, 2014.

COLMAN, Felicity. Affect. In: PARR, Adrian (Ed.). The Deleuze Dictionary. Edinburgh: Edinburgh University, 2010. p.11-13.

CVETKOVICH, Ann. Depression is Ordinary: Public Feelings and Saidiya Hartman's Lose Your Mother'. Feminist Theory, v.13, n. 2, p. 131-146, 2012.

DELEUZE, Gilles; GUATTARI, Félix. A thousand plateaus: Capitalism and schizophrenia. London: Continuum, 1980/2004.

DE RIVA MAYORAL, Silvia; REVELLES BENAVENTE, Beatriz. Hacia una pedagogía afectiva del movimiento. Tercio Creciente, n. 16, p. 7-30, 2019. Disponivel em: https://dx.doi. org/10.17561/rtc.n16.1. Acesso em: 11 fev. 2020.

DOMíNGUEZ, Enciso; LARA, Alí. Emociones y Ciencias Sociales en el s. XX: La precuela del Giro Afectivo. Athenea Digital, v. 14, n. 1, p. 263-288, 2014.

ERSTAD, Ola; SEFTON-GREEN, Julian. (ed.). Identity, Community, and Learning Lives in Digital Age. Cambridge: Cambridge University, 2013.

ESTEBAN, Mari Luz. Antropología del cuerpo. Género, itinerarios corporales, identidad y cambio. Barcelona: Bellaterra, 2004.

GROSZ, Elizabeth. Notes Towards a Corporeal Feminism. Australian Feminist Studies, v. 5, p. 1-15, 1987.

GROSZ, Elizabeth. Volatile Bodies: Toward a Corporeal Feminism. Bloomington: Indiana University, 1994.

GROSZ, Elizabeth. The Nick of Time: Politics, Evolution, and the Untimely. Durham: Duke University, 2004.

HERNÁNDEZ-HERNÁNDEZ, Fernando. La investigación basada en las artes. Propuestas para repensar la investigación en educación. Educatio Siglo XXI, n. 26, p. 85-118, 2008.

HERNÁNDEZ-HERNÁNDEZ, Fernando; SANCHO-GIL, Juana María; DOMINGOCOSCOLLOLA, María. Cartographies as spaces of inquiry to explore of teachers' nomadic learning trajectories. Digital Educational Review, n. 33, p. 105-119, 2018.

HERNÁNDEZ-HERNÁNDEZ, Fernando; REVELLES, Beatriz. La perspectiva post-cualitativa en la investigación educativa: genealogía, movimientos, posibilidades y tensiones, Educatio Siglo XXI, v. 37, n. 2, pp. 21-48, 2019. Disponivel em: http://dx.doi.org/10.6018/j/387001 Acesso em: 11 fev. 2020.

HICKEY-MOODY, Anna. Femifesta for Posthuman Art Education: Visions and Becomings. In: TAYLOR, C.; HUGHES, C. (eds.). Posthuman Research Practices in Education. London: Palgrave Macmillan, 2016. p. 258-266.

LARA, Alí. Teorías afectivas vintage. Apuntes sobre Deleuze, Bergson y Whitehead. Cinta moebio, n. 52, p. 17-36, 2015. 
LARA, Alí; DOMÍNGUEZ, Enciso. El Giro Afectivo. Athenea Digital, v. 13, n. 3, p. 101-119, 2013.

LATHER, Patti. Methodology-21: what do we do in the afterward? International Journal of Qualitative Studies in Education, v. 26, n. 6, p. 634-645, 2013.

LATHER, Patti. Top Ten + List: $(\mathrm{Re})$ Thinking Ontology in (Post)Qualitative Research.

Cultural Studies Critical Methodologies, v. 16, n, 2, p. 125-131. 2016. Disponivel em: http://dx.doi.org/10.1177/1532708616634734. Acesso em: 11 fev. 2020.

LATHER, Patti; ST. PIERRE, Elizabeth. Post-qualitative research. International Journal of Qualitative Studies in Education, v. 26, n. 6, p. 629-633, 2013.

LE BRETON, David. Sociología del cuerpo. Buenos Aires: Nueva visión, 2002.

MACPHAIL, Ann. Professional learning as a physical education teacher educator. Physical Education \& Sport Pedagogy, v. 16, n. 4, p. 435-451, 2011.

MARTíNEZ, Ana. La construcción social del cuerpo en las sociedades contemporáneas. Revista de Sociología, v. 73, p. 127-152, 2004. Disponivel em: http://dx.doi.org/10.5565/ rev/papers/v73n0.1111. Acesso em: 11 fev. 2020.

MASNY, Diana. Problematizing Qualitative Research: Reading a Data Assemblage with Rhizoanalysis. Qualitative Inquiry, v. 22, n. 8, p. 666-675, 2016. Disponivel em: http:// dx.doi.org/10.1177/1532708616636744. Acesso em: 11 fev. 2020.

MERLEAU-PONTY, Maurice. Phénoménologie de la perception. Paris: Gallimard, 1945.

MERLEAU-PONTY, Maurice. Lo visible y lo invisible. Madrid: Taurus. 1973.

MAZZEI, Lisa. A voice without organs: interviewing in posthumanist research. International Journal of Qualitative Studies in Education, v. 26, n. 6, p. 732-740, 2013. DOI 10.1080/09518398.2013.788761

NARDINI, Krizia. Embodied Thinking and the 'Transformative Matter' of (New) Feminist Materialist Theorizing. Artnodes, n.14, p.18-24, 2014.

PAULSTON, Rolland; LIEBMAN, Martin. An Invitation to Postmodern Social Cartography. Comparative Education Review, v. 38, n. 2, p. 215-232, 1994.

RICHARDS, Andrew; LEVESQUE-BRISTOL, Chantal. Student Learning and Motivation in Physical Education, Strategies: A Journal for Physical and Sport Educators, v. 27, n. 2, p. 43-46, 2014. DOI: $10.1080 / 08924562.2014 .879431$

RODRÍGUEZ, Rosana Paula. La vida encarnada: Significaciones sobre la experiencia corporal de las mujeres. Journal for Educators, Teachers and Trainers, v. 5, n.2, p. 115128, 2014.

ROGOWSKA-STANGRET, Monika. Corpor(e)al Cartographies of New Materialism: Meeting the Elsewhere Halfway. The Minnesota Review, v.88, n. 1, p, 59-68, 2017.

RUITENBERG, Claudia. Here be dragons: Exploring Cartography in Educational Theory and Research. Complicity: An International Journal of Complexity and Education, v.4, n. 1, p. 7-24, 2007.

ROGOFF, Irit. Academy as Potentiality. Zehar, n. 60/61, p. 4-9, 2006. 
SPINOZA, Baruch. Ética demostrada según el orden geométrico. Trad.Vidal Peña. Madrid: Orbis, 1980. (Original publicado en 1677).

TURNER, Bryan. Avances recientes en la teoría del cuerpo. REIS-Revista Española de Investigaciones Sociológicas, n. 68, p. 11-39, 1994.

ULMER, Jasmine B.; KORO-LJUNGBERG, Mirka. Writing Visually Through (Methodological) Events and Cartography. Qualitative Inquiry, v.21, n. 2, p. 138-152, 2015.

\section{Apoio:}

España. Ministerio de Economía, Empresa y Competitividad. EDU2015-70912-C2-1-R y EDU2015-70912-C2-2-R.

\section{Agradecimientos:}

Grupo de investigación ESBRINA-Subjetividades, visualidades y entornos educativos contemporáneos (2017SGR 12489. http://esbrina.eu) 